\title{
Random Quantum Operations
}

\author{
Wojciech Bruzda ${ }^{1}$, Valerio Cappellini ${ }^{1}$, Hans-Jürgen Sommers ${ }^{2}$, and Karol Życzkowski ${ }^{1,3}$ \\ ${ }^{1}$ Mark Kac Complex Systems Research Centre, Institute of Physics, \\ Jagiellonian University, ul. Reymonta 4, 30-059 Kraków, Poland \\ ${ }^{2}$ Fachbereich Physik, Universität Duisburg-Essen, Campus Duisburg, 47048 Duisburg, Germany \\ ${ }^{3}$ Centrum Fizyki Teoretycznej, Polska Akademia Nauk, Al. Lotników 32/44, 02-668 Warszawa, Poland
}

(Dated: October 13, 2008)

\begin{abstract}
We define a natural ensemble of trace preserving, completely positive quantum maps and present algorithms to generate them at random. Spectral properties of the superoperator $\Phi$ associated with a given quantum map are investigated and a quantum analogue of the Frobenius-Perron theorem is proved. We derive a general formula for the density of eigenvalues of $\Phi$ and show the connection with the Ginibre ensemble of real non-symmetric random matrices. Numerical investigations of the spectral gap imply that a generic state of the system iterated several times by a fixed generic map converges exponentially to an invariant state.
\end{abstract}

Random dynamical systems are subject of a considerable scientific interest. Certain statistical properties of classical deterministic systems which exhibit chaotic behavior can be described by random processes. A similar link exists also in the quantum theory, since quantum analogues of classically chaotic dynamical systems display spectral properties characteristic of ensembles of random matrices [1].

In the case of an isolated quantum system the dynamics is represented by a unitary evolution operator which acts on the space of pure quantum states. To characterize such evolution operators for periodically driven, quantum chaotic systems the Dyson circular ensembles of random unitary matrices are often used. The symmetry properties of the system determine which universality class should be used [2].

In a more general case of a quantum system coupled with an environment one needs to work with density operators. In such a case any discrete dynamics is governed by a quantum operation [3] which maps the set of all density operators of size $N$ into itself. Investigations of the set $\mathcal{S}_{\mathcal{N}}$ of all quantum operations play the key role in the field of quantum information processing [4], since any physical transformation of a state carrying quantum information has to be described by an element of this set.

To process quantum information one needs to transform a given quantum state in a controlled way. When designing a sequence of quantum operations, which constitutes a quantum algorithm, it is important to understand the properties of each operation and to estimate the influence of any imperfection in realization of an operation on the final result.

In order to describe an effect of external noise acting on a quantum system one often uses certain models of random quantum operations. On the other hand, random operations are purposely applied to obtain pseudo-random quantum circuits [5, 6]. These possible applications provide motivation for research on random quantum operations.

The main aim of this work is to construct a general class of random quantum operations and to analyze their properties. More formally, we introduce a natural probability measure which covers the entire set $\mathcal{S}_{N}$ of quantum operations and present an efficient algorithm to generate them randomly. We investigate spectral properties of superoperators associated with quantum operations and infer conclusions about the convergence of any initial state subjected to the repeated action of a given random operation to its invariant state. It is worth to emphasize that the spectral properties of superoperators are already accessible experimentally, as demonstrated by Weinstein et al. [7] in a study of a three-qubit nuclear magnetic resonance quantum information processor.

We start reviewing the classical problem, in which classical information is encoded into a probability vector $\vec{p}$ of length $N$. The set of all classical states of size $N$ forms the probability simplex $\Delta_{N-1}$. A discrete dynamics in this set is given by a transformation $p_{i}^{\prime}=S_{i j} p_{j}$, where $S$ is a real square matrix of size $N$, which is stochastic, i.e.:

a) $S_{i j} \geq 0$ for $i, j=1, \ldots, N$;

b) $\sum_{i=1}^{N} S_{i j}=1$ for all $j=1, \ldots, N$.

If a real matrix $S$ satisfies assumptions (a) and (b) then

i) the spectrum $\left\{z_{i}\right\}_{i=1}^{N}$ of $S$ belongs to the unit disk, $\left|z_{i}\right| \leq 1$, and the leading eigenvalue equals unity, $z_{1}=1$;

ii) the eigenspace associated with $z_{1}$ contains (at least) a real eigenstate $\vec{p}_{\text {inv }}$, which describes the invariant measure of $S$.

If additionally

c) $\sum_{j=1}^{N} S_{i j}=1$ for all $i=1, \ldots, N$

then the matrix $S$ is called bistochastic (doubly stochastic) and

iii) the maximally mixed state is invariant, $\vec{p}_{\mathrm{inv}}=(1 / N, \ldots, 1 / N)$. 
This is a form of the well-known Frobenius-Perron (FP) theorem and its proof may be found e.g. in [8, 9].

To generate a random stochastic matrix it is convenient to start with a square matrix $X$ from the complex Ginibre ensemble, all elements of which are independent complex random Gaussian variables. Then the random matrix

$$
S_{i j}:=\left|X_{i j}\right|^{2} / \sum_{m=1}^{N}\left|X_{m j}\right|^{2},
$$

is stochastic [10], and each of its columns forms an independent random vector distributed uniformly in the probability simplex $\Delta_{N-1}$.

Let us now discuss the quantum case, in which any state can be described by means of a positive, normalized operator, $\rho \geq 0, \operatorname{Tr} \rho=1$. Let $\mathcal{M}_{N}$ denote the set of all normalized states which act on a $N$-dimensional Hilbert space $\mathcal{H}_{N}$. A quantum analogue of a stochastic matrix is given by a linear quantum map $\Phi: \mathcal{M}_{N} \rightarrow \mathcal{M}_{N}$ which preserves the trace, and is completely positive (i.e. the extended map, $\Phi \otimes \mathbb{1}_{K}$, is positive for any size $K$ of the extension $\mathbb{1}_{K}$ ). Such a transformation is called quantum operation or stochastic map, and can be described by a matrix $\Phi$ of size $N^{2}$,

$$
\rho^{\prime}=\Phi \rho \quad \text { or } \quad \rho_{m \mu}^{\prime}=\Phi_{m \mu} \rho_{n \nu}
$$

It is convenient to reorder elements of this matrix [3, 11] defining the so-called dynamical matrix D,

$$
D(\Phi) \equiv \Phi^{R} \quad \text { so } \text { that } D_{\mu \nu}=\Phi_{n \nu}
$$

since the matrix $D$ is Hermitian if the map $\Phi$ preserves Hermiticity. Note that the above equation can be interpreted as a definition of the operation of reshuffling (realignment) of a matrix $X$, denoted by $X^{R}$. This definition is representation dependent, and corresponds to exchanging the position of some elements of a matrix. Obviously reshuffling is an involution: $D^{R}=\Phi$.

Due to the theorem of Choi [12], a map $\Phi$ is completely positive $(\mathrm{CP})$ if and only if the corresponding dynamical matrix $D$ is positive, $D \geq 0$. Hence $D$ can be interpreted as a positive operator acting on a composed Hilbert space $\mathcal{H}:=\mathcal{H}_{A} \otimes \mathcal{H}_{B}$. Any completely positive map can also be written in the Kraus form [13],

$$
\rho \rightarrow \rho^{\prime}=\sum_{i} A_{i} \rho A_{i}^{\dagger}
$$

The set of Kraus operators $A_{i}$ allows one to write down the linear superoperator as

$$
\Phi=\sum_{i} A_{i} \otimes \bar{A}_{i}
$$

where $\bar{A}_{i}$ is the complex conjugate of $A_{i}$, while $A_{i}^{\dagger}$ denotes the adjoint operator: $A_{i}^{\dagger}=\bar{A}_{i}^{T}$.

The map $\Phi$ is trace preserving, $\operatorname{Tr} \rho^{\prime}=\operatorname{Tr} \rho=1$, if $\sum_{i} A_{i}^{\dagger} A_{i}=\mathbb{1}_{N}$. This condition is equivalent to a partial trace condition imposed on the dynamical matrix,

$$
\operatorname{Tr}_{A} D(\Phi)=\mathbb{1}_{N}
$$

which implies $\operatorname{Tr} D=N$.

Since the dynamical map of an operation $\Phi$ is positive and normalized, the rescaled matrix $D / N$ may be considered as a state in an extended Hilbert space $\mathcal{H}_{A} \otimes \mathcal{H}_{B}$ of size $N^{2}$. Stochastic maps and states on the extended space $\mathcal{H}_{A} \otimes \mathcal{H}_{B}$ are related by the so-called Jamiotkowski isomorphism [3, 14] . Making use of the maximally entangled bipartite state $\left|\psi_{+}\right\rangle=\frac{1}{\sqrt{N}} \sum_{i=1}^{N}|i, i\rangle$, this can be expressed as $D(\Phi) / N=(\Phi \otimes \mathbb{1})\left|\psi_{+}\right\rangle\left\langle\psi_{+}\right|$. For completeness we sketch here a compact proof of the latter fact.

Proof. The projector $\left|\psi_{+}\right\rangle\left\langle\psi_{+}\right|$can be recasted in the form $\frac{1}{N} \sum_{i, j=1}^{N}|i\rangle\left\langle\left. j\right|_{A} \otimes \mid i\right\rangle\left\langle\left. j\right|_{B}\right.$, in which the matrix elements on $\mathcal{H}_{A}$ and $\mathcal{H}_{B}$ are explicit. Then, $\left(\Phi_{A} \otimes \mathbb{1}_{B}\right)\left|\psi_{+}\right\rangle\left\langle\psi_{+}\right|=\frac{1}{N} \sum_{i, j=1}^{N} \Phi(|i\rangle\langle j|)_{A} \otimes(|i\rangle\langle j|)_{B}$, which in turn can be expressed in coordinates as $\frac{1}{N} \sum_{i, j, \mu, \nu=1}^{N} \Phi_{\mu \nu}|\mu i\rangle\langle\nu j|$. Thus the result follows from definition (3) .

A quantum map is called unital if it leaves the maximally mixed state invariant. It is so if the Kraus operators satisfy $\sum_{i} A_{i} A_{i}^{\dagger}=\mathbb{1}_{N}$. The unitality condition may also be written in a form $\operatorname{Tr}_{B} D=\mathbb{1}_{N}$, dual to (6) . A CP quantum map which is trace preserving and unital is called bistochastic.

Spectral properties of positive operators were studied in the mathematical literature [15, 16] for a general framework of $C^{*}$-algebras. Here we analyze spectral properties of the operator $\Phi$ corresponding to a stochastic map and formulate a quantum analogue of the Frobenius-Perron theorem. 
Let $\Phi$ be a complex square matrix of size $N^{2}$, so that it represents an operator acting in a composite Hilbert space $\mathcal{H}_{N^{2}}=\mathcal{H}_{A} \otimes \mathcal{H}_{B}$. Let us order its complex eigenvalues according to their moduli, $\left|z_{1}\right| \geq\left|z_{2}\right| \geq \cdots \geq\left|z_{N^{2}}\right| \geq 0$.

Assume that $\Phi$ represents a stochastic quantum map, i.e. it satisfies

$\left.\left.a^{\prime}\right) \Phi^{R} \geq 0 ; \quad b^{\prime}\right) \sum_{k} \Phi_{i j}=\delta_{i j}$ so that $\operatorname{Tr}_{A} \Phi^{R}=\mathbb{1}$,

where the reshuffling operation, denoted by ${ }^{R}$, is defined in eq. (3). Then

$\left.i^{\prime}\right)$ the spectrum $\left\{z_{i}\right\}_{i=1}^{N^{2}}$ of $\Phi$ belongs to the unit disk, $\left|z_{i}\right| \leq 1$, and the leading eigenvalue equals unity, $z_{1}=1$,

$\left.\mathrm{ii}^{\prime}\right)$ one of the corresponding eigenstates forms a matrix $\omega$ of size $N$ which is positive, normalized $(\operatorname{Tr} \omega=1)$ and is invariant under the action of the map, $\Phi(\omega)=\omega$.

If additionally

$\left.c^{\prime}\right) \operatorname{Tr}_{B} \Phi^{R}=\mathbb{1}$, then the map is called bistochastic and

iii') the maximally mixed state is invariant, $\omega=\mathbb{1} / N$.

Proof. Assumption $\left(\mathrm{a}^{\prime}\right)$ implies that the quantum map is completely positive while $\left(\mathrm{b}^{\prime}\right)$ implies the trace preserving property. Hence $\Phi$ is a linear map which sends the convex compact set $\mathcal{M}_{N}$ of mixed states into itself. Due to the Schauder fixed-point theorem [17] such a transformation has a fixed point - an invariant state $\omega \geq 0$ such that $\Phi \omega=\omega$. Thus $z_{1}=1$ and all eigenvalues fulfil $\left|z_{i}\right| \leq 1$, since otherwise the assumption that $\Phi$ maps the compact set $\mathcal{M}_{N}$ into itself would be violated.

The spectral properties of stochastic maps are similar to those of classical stochastic matrices discussed in [18]. As noted in [19, 20] the spectrum of the superoperator is symmetric with respect to the real axis - see Fig. 1. Such spectra for a quantum map corresponding to a classically chaotic irreversible system where studied in [21].

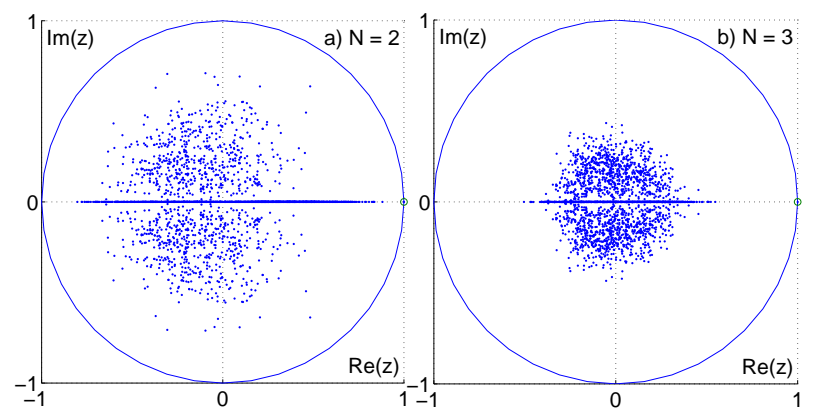

FIG. 1: Spectra of an ensemble of random superoperators $\Phi$ : a) 800 operations for $N=2$ and b) 300 operations for $N=3$.

Let us analyze briefly some particular cases of quantum operations. For any unitary rotation, $\Phi(\rho)=U \rho U^{\dagger}$ the Kraus form (44) consists of a single operator only, $A_{1}=U$. Thus according to (5) the superoperator is given by a unitary matrix $\Phi=U \otimes \bar{U}$ of size $N^{2}$. Denote the eigenphases of $U$ by $\alpha_{i}$ where $i=1, \ldots N$. Then the spectrum of $\Phi$ consists of $N^{2}$ phases given by $\alpha_{i}-\alpha_{j}$ for $i, j=1, \ldots, N$. All diagonal phases for $i=j$ are equal to zero, hence the leading eigenvalue of the superoperator $\Phi, z_{1}=1$, exhibits multiplicity not smaller than $N$.

Consider now a quantum map for which $\Phi^{R}$ is diagonal. This special case can be treated as classical, since then $\Phi$ describes a classical dynamics in $\Delta_{N-1}$, while the generalized quantum version of the FP theorem reduces to its standard version. Reshaping a diagonal dynamical matrix of size $N^{2}$ one obtains then a matrix $S$ of size $N$, where $S_{i j}=\Phi_{i j}$, (no summation performed!). Then assumption $\left(\mathrm{a}^{\prime}\right)$ (all diagonal elements of $\Phi^{R}$ are positive) gives (a), while the trace preserving condition $\left(\mathrm{b}^{\prime}\right)$ implies the probability preserving condition (b). Similarly, the additional condition $\left(\mathrm{c}^{\prime}\right)$ for quantum unitality gives condition (c) which imposes that the uniform vector is invariant under multiplication by a bistochastic matrix $S$. Similarly, conclusions (i'), (ii') and (iii') of the quantum version of the theorem imply conclusions (i-iii) of the standard (classical) Frobenius-Perron theorem.

We are interested in defining an ensemble of random operations [3]. A simple choice of random external fields [22], defined as a convex combination of an arbitrary number $k$ of unitary transformations, $\rho^{\prime}=\sum_{i=1}^{k} p_{i} V_{i} \rho V_{i}^{\dagger}$, produces bistochastic operations only.

Let us then consider first a method of constructing random states by $N \times M$ rectangular random complex matrices of the Ginibre ensemble [23]. Taking

$$
\rho:=X X^{\dagger} / \operatorname{Tr} X X^{\dagger}
$$


we get a positive normalized state $\rho$. For $M=1$ we obtain a recipe to generate random pure states, while for $M=N$ the measure induced by the Ginibre ensemble coincides with the Hilbert-Schmidt measure in $\mathcal{M}_{N}[24]$ and the average purity, $\left\langle\operatorname{Tr} \rho^{2}\right\rangle_{\mathrm{HS}}$, scales as $1 / N[3,10]$.

Here we propose an analogous algorithm of constructing a random operation:

1) fix $M \geq 1$ and take a $N^{2} \times M$ random complex Ginibre matrix $X$;

2) find the positive matrix $Y:=\operatorname{Tr}_{\mathrm{A}} X X^{\dagger}$ and its square root $\sqrt{Y}$;

3) write the dynamical matrix (Choi matrix)

$$
D=\left(\mathbb{1}_{N} \otimes \frac{1}{\sqrt{Y}}\right) X X^{\dagger}\left(\mathbb{1}_{N} \otimes \frac{1}{\sqrt{Y}}\right)
$$

4) reshuffle the Choi matrix according to (3) to obtain the superoperator $\Phi=D^{R}$, and use it as in (2) to produce a random map.

It is not difficult to check that the relation (6) holds due to (8), so the random map preserves the trace. Such a renormalization to obtain the Choi matrix was independently used in [25]. This method is simple to apply for numerical simulations, and exemplary spectra obtained in the case $M=N^{2}$ are shown in Fig.1.

For larger $N$, the subleading eigenvalue modulus $r=\left|z_{2}\right|$ is smaller, so the convergence rate of any initial $\rho_{0}$ to the invariant state $\omega$ occurs faster. To demonstrate this effect we studied the decrease of an average trace distance in time, $L(t)=\left\langle\operatorname{Tr}\left|\Phi^{t}\left(\rho_{0}\right)-\omega\right|\right\rangle_{\psi}$, where the average is performed over an ensemble of initially pure random states, $\rho_{0}=|\psi\rangle\langle\psi|$. Numerical results confirm an exponential convergence, $L(t) \sim \exp (-\alpha t)$. The mean convergence rate $\langle\alpha\rangle_{\Phi}$, averaged over an ensemble of random operations, increases with the dimension $N$ like log $N$, with slope very close to unity - see Fig. 2.
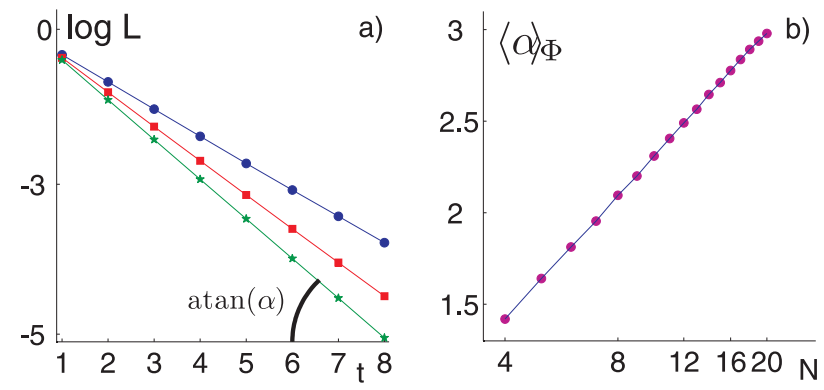

FIG. 2: a) Average trace distance of random pure states to the invariant state of $\Phi$ as a function of time for $N=4(\bullet), 6(\boldsymbol{\square}), 8(\star)$; b) mean convergence rate $\langle\alpha\rangle_{\Phi}$ as a function of the system size $N$, plotted in log scale.

To explain these findings we need to analyze spectral properties of an ensemble of random operations. Consider first a random Choi matrix $D$ obtained by the above algorithm from a Ginibre matrix $X$, generated according to the distribution $\propto \exp \left(-\operatorname{Tr} X X^{\dagger}\right)$. Then $D$ is of the Wishart type and has the distribution

$$
P(D) \propto \int d X \exp \left(-\operatorname{Tr} X X^{\dagger}\right) \delta\left(D-W X X^{\dagger} W^{\dagger}\right)
$$

where $W:=\mathbb{1} \otimes\left(\operatorname{Tr}_{A} X X^{\dagger}\right)^{-1 / 2}$. This integral can be rewritten with help of another Delta function,

$$
P(D) \propto \int d Y \int d X \exp (-\operatorname{Tr} Y) \delta\left(D-W X X^{\dagger} W^{\dagger}\right) \delta\left(Y-\operatorname{Tr}_{A} X X^{\dagger}\right) .
$$

Using the Delta function property and taking the Jacobians into account we arrive at

$$
P(D) \propto \operatorname{det}\left(D^{M-N^{2}}\right) \delta\left(\operatorname{Tr}_{\mathrm{A}} D-\mathbb{1}\right)
$$

which shows that there are no other constraints on the distribution of the Choi matrix, besides the partial trace condition and positivity. This is equivalent to saying that the $M$ Kraus matrices $A_{i}$, which form a map $\Phi$, constitute a $(M N) \times N$ truncated part of a unitary matrix $U$ of size $N M$. A natural assumption is that the matrix $U$ is distributed according to the Haar measure. Unitarity constraints become weak for large $N$, so the non-Hermitian $N \times N$ truncations $A_{i}$ are described by the complex Ginibre ensemble 23]: Their spectra cover uniformly the disk of radius $1 / \sqrt{N}$ in the complex plane [26]. 
Therefore we are in position to present an alternative algorithm of generating the same ensemble of random operations, which has a simple physical interpretation:

$\left(1^{\prime}\right)$ Choose a random unitary matrix $U$ according to the Haar measure on $U(N M)$;

$\left(2^{\prime}\right)$ Construct a random map defined by

$$
\rho^{\prime}=\operatorname{Tr}_{M}\left[U(\rho \otimes|\nu\rangle\langle\nu|) U^{\dagger}\right]
$$

Hence this random operation corresponds to an interaction with an $M$-dimensional environment, initially in a random pure state $|\nu\rangle$. Of a special importance is the case $M=N^{2}$, for which the term with the determinant in (11) disappears, so the matrices $D$ are generated according to the measure analogous to the Hilbert-Schmidt measure. This case provides thus generic dynamical matrices of a full rank and can be recommended for numerical implementation.

To analyze spectral properties of a superoperator $\Phi$ let us use the Bloch representation of a state $\rho$,

$$
\rho=\sum_{i=0}^{N^{2}-1} \tau_{i} \lambda^{i}
$$

where $\lambda^{i}$ are generators of SU(N) such that $\operatorname{tr}\left(\lambda^{i} \lambda^{j}\right)=\delta^{i j}$ and $\lambda^{0}=\mathbb{1} / \sqrt{N}$. Since $\rho=\rho^{\dagger}$, the generalized Bloch vector $\vec{\tau}=\left[\tau_{0}, \ldots, \tau_{N^{2}-1}\right]$, also called coherence vector, is real. Thus the action of the map $\Phi$ can be represented as

$$
\tau^{\prime}=\Phi(\tau)=C \tau+\kappa
$$

where $C$ is a real asymmetric contraction matrix of size $N^{2}-1$ while $\vec{\kappa}$ is a translation vector, which vanishes for bistochastic maps. Their elements can be expressed in terms of the Kraus operators, e.g. $C_{i j}=\operatorname{Tr} \sum_{k} \lambda^{i} A_{k} \lambda^{j} A_{k}^{\dagger}$, while $\kappa_{i}=\operatorname{Tr} \lambda^{i} \lambda^{0} \sum_{k} \tau_{k} A_{k}^{\dagger}$. Thus there exists a real representation of the superoperator

$$
\Phi=\left[\begin{array}{ll}
1 & 0 \\
\kappa & \mathrm{C}
\end{array}\right] \text {. }
$$

Eigenvalues of $C$, denoted by $\left\{\Lambda_{i}\right\}_{i=1}^{N^{2}-1}$, are also eigenvalues of $\Phi$. We are going to study the case $M=N^{2}$, for which the distribution (11) simplifies. Like for the real Ginibre ensemble [27, 28] one may derive in this case the measure in the space of eigenvalues $\Lambda_{i}$ of $C$

$$
d \mu(\Lambda)=\left|\prod_{i} d \Lambda_{i}\right| \prod_{k<l}\left|\Lambda_{k}-\Lambda_{l}\right| G(\Lambda)
$$

where $G(\Lambda)$ is given by the distribution of (real) traces,

$$
G(\Lambda):=\left\langle\prod_{\nu=1}^{N^{2}-1} \delta\left(\sum_{i=1}^{N^{2}-1}\left(\Lambda_{i}\right)^{\nu}-\operatorname{Tr} C^{\nu}\right)\right\rangle
$$

with the average

$$
\langle f(C)\rangle:=\int d C d \kappa \Theta(D \geqslant 0) f(C) .
$$

The domain of integration is given by the conditions for complete positivity, $D \geq 0$, which is not easy to work with, even for $N=2$. For large $N$ we can expect that these conditions do not play an important role, so the dependence $G(\Lambda)$ is weak, and the measure for $C$ can be described by the real Ginibre ensemble of non-symmetric Gaussian matrices. The spectrum of such random matrices consists of a component on the real axis, the probability density of which is given asymptotically by the step function $P(x)=\frac{1}{2} \Theta(1-|x|)$ [29, 30], while remaining eigenvalues cover uniformly the unit circle according to the Girko distribution [1].

To analyze the spectra of random operators $\Phi$ one needs to set the scale. The mean purity of a random state $\sigma$ of size $N^{2}$ behaves as $N^{-2}[10]$ and $D=N \sigma$, thus the average $\operatorname{Tr} D^{2}=\operatorname{Tr} \Phi \Phi^{\dagger}$ is of the order of unity. Hence, the rescaled matrix $\Phi^{\prime}:=N \Phi$ of size $K=N^{2}$ has the normalization $\operatorname{Tr} \Phi^{\prime}\left(\Phi^{\prime}\right)^{\dagger} \approx K$, which assures that the radius of the circle is equal to unity.

Thus we arrive at the following conjecture: for large $N$ the statistical properties of a rescaled random superoperator $N \Phi$ are described by the real Ginibre ensemble. We confirmed this conjecture by a detailed numerical investigation. Fig. 3 shows the density of complex eigenvalues of random superoperators for $N=10$ with $M=N^{2}$ and the 


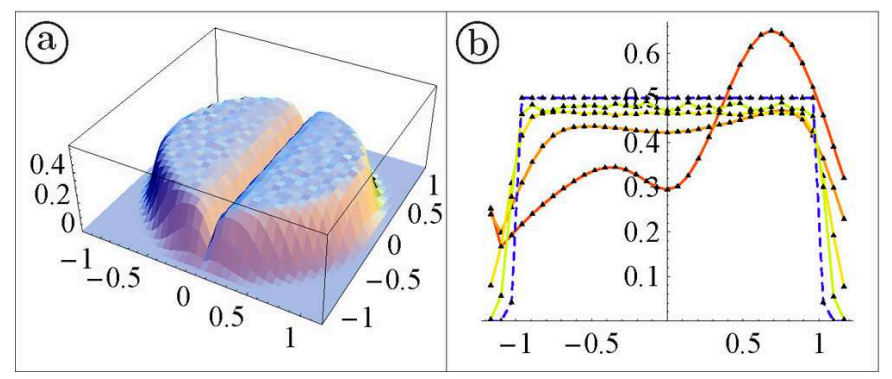

FIG. 3: a) Distribution of complex eigenvalues of $10^{4}$ rescaled random operators $\Phi^{\prime}$ already for $N=10$ can be approximated by the circle law. b) Distribution of real eigenvalues $P(x)$ of $\Phi^{\prime}$ plotted for $N=2,3,7$ and 14 tends to the step function, characteristic of real Ginibre ensemble.

distribution $P(x)$ of the real eigenvalues. As the spectrum of the rescaled operator $\Phi^{\prime}=N \Phi$ tends to be localized in the unit circle, we infer that the size of the subleading eigenvalue $r=\left|z_{2}\right|$ of $\Phi$ behaves as $1 / N$, hence the convergence rate $\alpha$ scales as $\ln N$.

Numerical studies were also performed for random maps acting on states of a fixed dimension $N$. In this case the subleading eigenvalue of a random map (12) decreases with the varying size of the environment $M$ as $r \sim 1 / \sqrt{M}$. Similar investigations were also performed under a constraint that the dynamical matrix $D$ is diagonal. In this case the assumption $M=1$ allows to obtain a random stochastic matrix $S$ of size $N$, such that each of its columns is generated independently with respect to the flat measure in the $(N-1)$ dimensional simplex of probability distributions. Analyzing the average trace of $S S^{T}$ we infer that in this case the complex spectrum can be described by the Girko distribution, which covers uniformly the disk of radius $r \sim 1 / \sqrt{N}$. These spectral properties of random stochastic matrices, confirmed by our numerical results, were rigorously analyzed in a recent paper of Horvat [31].

In this work we analyzed superoperators associated with quantum stochastic maps and their spectral properties and formulated a quantum analogue of the Frobenius-Perron theorem. We defined an ensemble of random operations, presented an explicit algorithm to generate them, and showed an exponential convergence of a generic state of the system to the invariant state under subsequent action of a fixed map. We demonstrated that for a large dimension of the Hilbert space, used to describe quantum dynamics, the spectral properties of a generic superoperator can be described by the Ginibre ensemble of real random matrices.

It is a pleasure to thank M.D. Choi, W. Słomczyński and J. Zakrzewski for helpful remarks. We acknowledge financial support by the SFB Transregio-12 project, the European grant COCOS, and a grant number N202 099 31/0746 of Polish Ministry of Science and Education.

[1] M. L. Mehta Random Matrices, III ed. (Academic, New York, 2004).

[2] F. Haake Quantum Signatures of Chaos, II ed. (Springer, Berlin, 2006)

[3] I. Bengtsson and K. Życzkowski, Geometry of Quantum States, (Cambridge University Press, Cambridge, 2006).

[4] M. A. Nielsen and I. L. Chuang, Quantum Computation and Quantum Information, (Cambridge University Press, Cambridge, 2000).

[5] J. Emerson, E. Livine, and S. Lloyd, Phys. Rev. A 72, 060302(R) (2005);

[6] W. G. Brown Y. S. Weinstein, and L. Viola, preprint arXiv:0802.2675

[7] Y.S. Weinstein, T.F. Havel, J. Emerson, N. Boulant, M. Saraceno M, S. Lloyd and D.G. Cory J. Chem. Phys. 121, 6117 (2004)

[8] A. W. Marshall and I. Olkin, The Theory of Majorizations and Its Applications (Academic Press, New York, 1979)

[9] D. S. Bernstein, Matrix mathematics: theory, facts, and formulas with application to linear systems theory, Princeton University Press, Princeton, 2005.

[10] K. Życzkowski and H.-J. Sommers, J. Phys. A: Math. Gen. 34(35), 7111-7125 (2001).

[11] E. C. G. Sudarshan, P.M Mathews, and J. Rau, Phys. Rev. 121, 920 (1961).

[12] M.-D. Choi, Linear Alg. Appl. 10, 285 (1975).

[13] K. Kraus, Ann. Phys. 64, 311 (1971).

[14] A. Jamiołkowski, Rep. Math. Phys. 3, 275 (1972).

[15] D. Evans and R. Høegh-Krohn, J. London Math. Soc. 17345 (1978).

[16] U. Groh, Linear Algebra Appl. 42213 (1982).

[17] J. Schauder, Studia Math. 2, 171-180 (1930).

[18] K. Życzkowski, M. Kuś, W. Słomczyński and H.-J. Sommers, J. Phys. A 36, 3425-3450 (2003). 
[19] P. Pepłowski and F. Haake, J. Phys. A 26, 2473 (1993)

[20] B. M. Terhal and D. DiVincenzo, Phys. Rev. A61, 22301 (2000).

[21] A. Łoziński, P. Pakoński and K. Życzkowski, Phys. Rev. E 66, 065201(R4) (2002).

[22] R. Alicki and K. Lendi, Quantum Dynamical Semigroups and Their Applications, (Springer, Berlin, 1987)

[23] J. Ginibre, J. Math. Phys. 6, 440 (1965).

[24] H.-J. Sommers and K. Życzkowski, J. Phys. A 37, 8457 (2004).

[25] K. M.R. Audenaert and S. Scheel, N. J. Phys. 10, 023011 (2008).

[26] K. Życzkowski and H.-J. Sommers, J. Phys. A 33, 2045 (2000).

[27] N. Lehmann and H.-J. Sommers, Phys. Rev. Lett. 67, 941 (1991).

[28] H.-J. Sommers and W. Wieczorek, J. Phys. A 41405003 (2008).

[29] H.-J. Sommers J. Phys. A 40, F671 (2007);

[30] P.J. Forrester and T. Nagao, Phys. Rev. Lett. 99, 050603 (2007).

[31] M. Horvat, preprint, Lubliana 2008. 